Original

\title{
Comparison of salivary hemoglobin measurements for periodontitis screening
}

\author{
Ayako Okada1), Yoshiaki Nomura1), Kaoru Sogabe1), Hirofumi Oku²), \\ Arika Sato Gillbreath ${ }^{3)}$, Fumihiko Hino4), Hideo Hayashis), Hirokazu Yoshino ${ }^{3)}$, \\ Hisanori Utsunomiya4), Kazuyuki Suzuki5), Keizo Koresawa4), Kenji Koba3), \\ Kimiyuki Uetani3), Mami Kotoh3), Naoyuki Nishitsuji3), Satoshi Akutsu6), \\ Takakazu Nakasone3), Yasushi Tobi6), Yoichi Fukuzawa3), Yoshihide Yabuki3), \\ Yoshinobu Naono4), Masataka Yajima ${ }^{3)}$, Keita Shimizu${ }^{4)}$, and Nobuhiro Hanada1) \\ 1)Department of Translational Research, School of Dental Medicine, Tsurumi University, Yokohama, Japan \\ 2)Shikokuchuken Co., Ltd, Kagawa, Japan \\ ${ }^{3)}$ Tokyo Minato-ku Shiba Dental Association, Tokyo, Japan \\ 4)Ehime Dental Association, Matsuyama, Japan \\ 5)Alfresa Pharma Corporation, Osaka, Japan \\ 6)MKT Department-II, Marketing Office, Sales Division, Eiken Chemical Co., Ltd., Tokyo, Japan
}

(Received March 9, 2016; Accepted July 12, 2016)

\begin{abstract}
Salivary hemoglobin (Hb) for screening of periodontitis is approved under the pharmaceutical affairs law of Japan. Two reagents are commercially available for the modified fecal occult blood test: Saliva Hemo Plus and OC-AUTO S Latex Reagent. We simultaneously measured split specimens from 561 samples by using these two methods and compared the differences and agreement between both methods. Moreover, saliva samples were collected from 10 subjects at five time points during the day for analysis of circadian variations and fluctuation. The Pearson's correlation coefficient for these two reagents was 0.794. The Bland-Altman plot of differences in salivary $\mathrm{Hb}$ levels measured by the two reagents indicated that the difference included fixed errors $(0.55$ $\mu \mathrm{g} / \mathrm{mL}$ ). On analysis of circadian variations, no statistically significant differences were observed using the

Correspondence to Dr. Nobuhiro Hanada, Department of Translational Research, School of Dental Medicine, Tsurumi University, 2-1-3 Tsurumi, Tsurumi-ku, Yokohama, Kanagawa 230-8501, Japan

Fax: +81-45-573-2473 E-mail: hanada-n@tsurumi-u.ac.jp

doi.org/10.2334/josnusd.16-0204

DN/JST.JSTAGE/josnusd/16-0204
\end{abstract}

Friedman test. However, fixed errors were observed between wake-up time and before dinner and before lunch and before dinner, and no random errors were observed by Bland-Altman analysis. In conclusion, the salivary Hb levels measured using OC-AUTO S Latex Reagent were lower than those measured using Saliva Hemo Plus, along with a tendency for higher levels in the morning. Thus, when performing salivary tests these observations must be considered.

Keywords: periodontal disease; salivary hemoglobin; saliva test.

\section{Introduction}

Periodontal disease is one of the most prevalent oral diseases among middle-aged and elderly people. Screening for periodontal disease plays an important role in its prevention and in improving oral health-related quality of life.

Conventionally, screening for periodontal disease is performed by pocket probing of representative teeth by dental professionals. However, pocket probing is associated with fundamental biological issues because patients 
with periodontitis are at a greater risk of bacteremia due to periodontal probing (1).

Several salivary biomarkers have been suggested to be correlated with periodontal conditions $(2,3)$. These markers have been investigated for use in clinical diagnosis or screening. In our previous study, we reported that salivary lactate dehydrogenase had the highest sensitivity and specificity for periodontal disease (with at least one probing depth $>4 \mathrm{~mm}$ ) compared with other biochemical markers such as aspartate aminotransferase, alanine aminotransferase, alkaline phosphatase, creatinine, and blood urea nitrogen (4). However, salivary lactate dehydrogenase is not approved under the pharmaceutical affairs law in Japan for screening of periodontal disease as an in vitro diagnostic test; in addition, its sensitivity and specificity were $<0.7$. In contrast, salivary hemoglobin $(\mathrm{Hb})$ is the only approved test. At an earlier stage, salivary $\mathrm{Hb}$ has been measured by colorimetric detection, and only free $\mathrm{Hb}$ (f-Hb) has been measured (4-6). Reportedly, the sensitivity and specificity of $\mathrm{f}-\mathrm{Hb}$ were 0.81 and 0.27 , respectively (4). This method revealed that contamination of blood from other species in food was detected. Therefore, the diagnostic precision of $\mathrm{f}-\mathrm{Hb}$ in saliva was insufficient. Subsequently, methods for salivary $\mathrm{Hb}$ measurement have been improved. Two reagents based on the modified fecal occult blood tests are now commercially available, and these reagents use polyclonal antibodies, which are conjugated with colloidal gold (Saliva Hemo Plus, Alfresa Pharma Corporation, Osaka, Japan) or latex particles (OC-AUTO S Latex Reagent, Eiken Chemical Co., Ltd., Tokyo, Japan). The use of these methods increases the sensitivity of salivary $\mathrm{Hb}$ detection (7-9) and also improves sensitivity and specificity. Salivary $\mathrm{Hb}$ levels measured using Saliva Hemo Plus revealed a $>0.7$ sensitivity and specificity for bleeding (8). In addition, sensitivity and specificity of salivary $\mathrm{Hb}$ measured by OC-AUTO S Latex Reagent were also $>0.7$, according to the criteria of the Center for Disease Control and Prevention in partnership with the American Academy of Periodontology (10). In terms of likelihood ratio, the likelihood ratio for Saliva Hemo Plus, OC-AUTO S Latex Reagent, and f-Hb were 3.33, 3.22 , and 1.42 , respectively.

In order to promote saliva testing for screening of periodontal disease, basic data for validation are necessary. In this study, we simultaneously measured a single sample by using two methods and compared their differences and agreement. In addition, circadian variations and fluctuations during the day were assessed.

\section{Materials and Methods \\ Study population}

The study population was selected from subjects who registered for oral health promotional events sponsored by two dental associations (Ehime Dental Association and Minato-ku Shiba Dental Association). Inclusion criteria were adults aged $>20$ years with at least 20 teeth remaining and without dentures. In total, 561 subjects (410 male and 151 female) were selected (age: $54.8 \pm$ 14.05 years).

The study population for analysis of circadian variations and fluctuations during the day was selected from the subjects who worked at Shikokuchuken Co. Ltd. Inclusion criteria were adults aged $>20$ years with at least 20 teeth remaining and without any dentures. In total, 10 subjects (six male and four female) were selected (age: $40.72 \pm 9.06$ years) .

This study was approved by the Ethics Committee of Tsurumi University, School of Dental Medicine (approval number: 430), and was conducted in accordance with the Helsinki 1964 Declaration.

\section{Measuring salivary $\mathbf{H b}$}

Stimulated whole saliva was collected by chewing on a gum base containing neither fragrant nor taste ingredients for five min. To examine salivary Hb levels, 100 $\mu \mathrm{L}$ of the collected saliva was immediately transferred into a diluent solution with preserving agents and kept at $4^{\circ} \mathrm{C}$. These samples were immediately transported to the medical examination laboratory. Salivary $\mathrm{Hb}$ levels were measured using Saliva Hemo Plus (Alfresa Pharma Corporation) and OC-AUTO S Latex Reagent (Eiken Chemical Co., Ltd.), according to the manufacturer's instructions (8-10).

For evaluation of circadian variations and fluctuations during the day, saliva samples were collected at five different time points: wake up, before lunch, 2 $\mathrm{h}$ after lunch, before dinner, and before bedtime. After confirming that the subjects had not consumed any food for at least $1 \mathrm{~h}$, stimulated whole saliva was collected by chewing a gum base containing neither fragrant nor taste ingredients for $5 \mathrm{~min}$. To examine salivary $\mathrm{Hb}$ levels, 100 $\mu \mathrm{L}$ of the collected saliva was immediately transferred into a diluent solution with preserving agents and stored at $4^{\circ} \mathrm{C}$. Salivary $\mathrm{Hb}$ levels were measured using Saliva Hemo Plus, according to the manufacturer's instructions.

\section{Statistical analysis}

In order to assess correlations between salivary $\mathrm{Hb}$ levels measured under different conditions, Pearson's correlation coefficient and linear regression formula were 
calculated. $P$ values of cross tabulations were calculated using chi-squared test and Fisher's exact test when counts were $<5$ per cell. $P$ values for circadian variations were determined using the Friedman test. These statistical analyses were performed using IBM Statistical Package for the Social Sciences Statistics Ver 19.0 (IBM SPSS, Tokyo, Japan). To identify any systemic errors, BlandAltman analysis was conducted (11-17). Differences in the two values were evaluated by means and $95 \%$ confidence intervals (CIs). The random errors were evaluated using the test of no correlation of mean and difference of the two values. This analysis was performed using S-Plus software Ver 6.0 (NTT DATA, Tokyo, Japan).

\section{Results}

Figure 1A presents a scatter plot of results for the 561 samples evaluated using the two reagents. Pearson's correlation coefficient was 0.794 , and the formula for linear regression was $\mathrm{Y}=1.691 \mathrm{X}-5.907$. Pearson's correlation coefficient and the regression formula were affected by certain samples with high values. Overall, 393 samples $(70.0 \%, 393 / 561)$ presented values $<10$ $\mu \mathrm{g} / \mathrm{mL}$ with both reagents, and the screening level of salivary $\mathrm{Hb}$ for periodontitis is $2.0 \mu \mathrm{g} / \mathrm{mL}$. Figure 1B presents a magnified scatter plot for samples $<10 \mu \mathrm{g} / \mathrm{mL}$. Hence, the linear regression formula was changed to $\mathrm{Y}=$ $0.7872 \mathrm{X}-0.0408$.

To identify any systemic errors between salivary $\mathrm{Hb}$ levels calculated using these two reagents, Bland-Altman analysis was conducted for the aforementioned 393 samples. Figure 2 presents a Bland-Altman plot of differences in the salivary $\mathrm{Hb}$ levels measured using the two reagents against the mean values measured using the two reagents. The mean value of differences and $95 \%$ CI was 0.29 (95\% CI\%: 0.18-0.40); moreover, by performing the test of no correlation, a $P$ value of 0.869 was obtained. Therefore, the difference included fixed errors and not random errors. This result indicated that salivary $\mathrm{Hb}$ levels measured using OC-AUTO S Latex Reagent were lower by $0.18-0.40 \mu \mathrm{g} / \mathrm{mL}$ than those measured using Saliva Hemo Plus. The optimal cutoff value of the OC-AUTO S Latex Reagent was calculated using ROC analysis with a cutoff point of $2.0 \mu \mathrm{g} / \mathrm{mL}$ of the OC-AUTO S Latex Reagent as a standard. The optimal cutoff point that showed the highest crude hit rate for the OC-AUTO S Latex Reagent was $1.45 \mu \mathrm{g} / \mathrm{mL}$.

The cross tabulation for positive and negative results for the two reagents is presented in Table 1. Cross tabulation when the cutoff point for OC-AUTO S Latex Reagent was set as 1.45 is presented in Table 1B. The crude hit rate improved from 89.3 to $90.2 \%$.

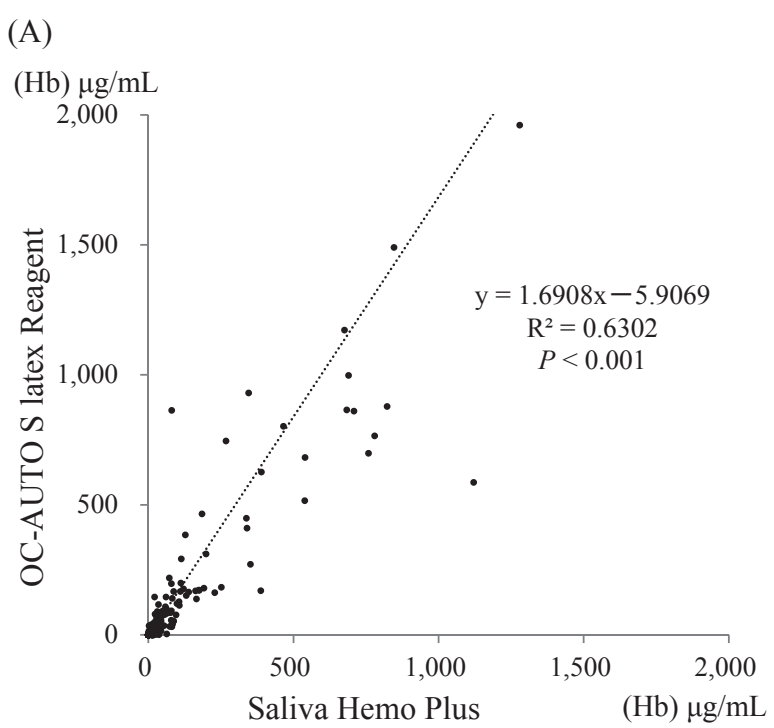

(B)

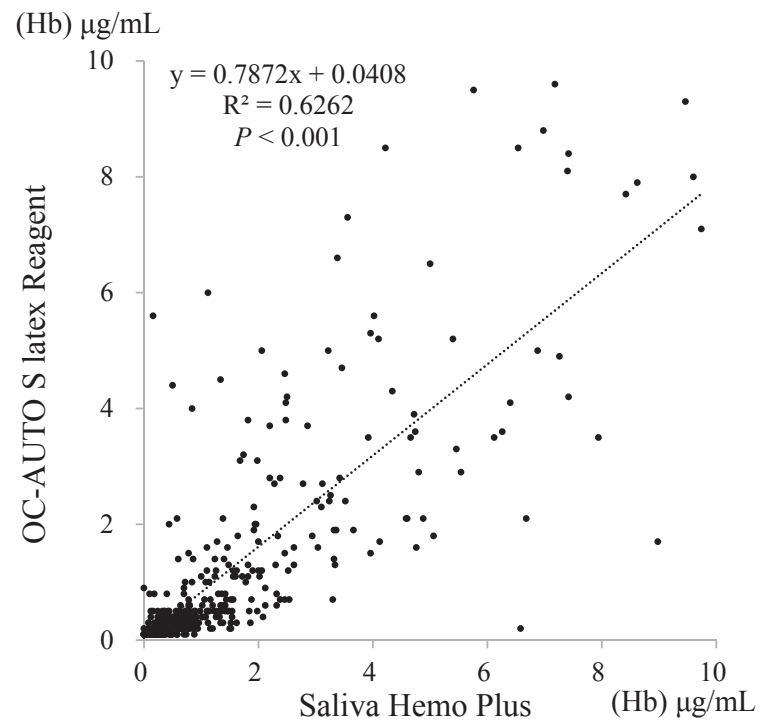

Fig. 1 Scatter plot of salivary hemoglobin $(\mathrm{Hb})$ levels measured using the two reagents. A) All samples investigated in this study. B) Samples with salivary $\mathrm{Hb}$ levels $<10 \mu \mathrm{g} / \mathrm{mL}$.

To confirm the stability of salivary Hb levels, samples provided by the Ehime Dental Association were measured at one and three days after sampling. Scatter plots for salivary $\mathrm{Hb}$ levels by using Saliva Hemo Plus and OC-AUTO S Latex Reagent are shown in Fig. 3A and $\mathrm{B}$, respectively. Pearson's correlation coefficients were 0.993 for Saliva Hemo Plus and 0.992 for OC-AUTO S Latex Reagent. By conducting Bland-Altman analysis of the 50 samples with salivary $\mathrm{Hb}$ levels $<10 \mu \mathrm{g} / \mathrm{mL}$, the differences did not include fixed or random errors. The means and $P$ values of the differences were 0.163 (95\% CI: $0.001-0.319 ; P=0.269)$ for Saliva Hemo Plus and 0.198 (95\% CI: $0.001-0.392 ; P=0.559$ ) for OC-AUTO 
Table 1 Cross tabulation of positive and negative test results for the two reagents (A)

\begin{tabular}{lcccc}
\hline & & \multicolumn{2}{c}{ OC-AUTO S Latex Reagent } & \multirow{2}{*}{$P$} \\
\cline { 2 - 4 } & & $<2.0 \mu \mathrm{g} / \mathrm{mL}$ & $\geq 2.0 \mu \mathrm{g} / \mathrm{mL}$ & $<0.001$ \\
\hline Saliva Hemo Plus & $<2.0 \mu \mathrm{g} / \mathrm{mL}$ & 282 & 12 & \\
& $\geq 2.0 \mu \mathrm{g} / \mathrm{mL}$ & 48 & 219 & \\
\hline
\end{tabular}

\begin{tabular}{|c|c|c|c|c|}
\hline & & \multicolumn{2}{|c|}{ OC-AUTO S Latex Reagent } & \multirow{2}{*}{$P$} \\
\hline & & $<1.45 \mu \mathrm{g} / \mathrm{mL}$ & $\geq 1.45 \mu \mathrm{g} / \mathrm{mL}$ & \\
\hline \multirow[t]{2}{*}{ Saliva Hemo Plus } & $<2.0 \mu \mathrm{g} / \mathrm{mL}$ & 272 & 22 & $<0.001$ \\
\hline & $\geq 2.0 \mu \mathrm{g} / \mathrm{mL}$ & 33 & 234 & \\
\hline
\end{tabular}

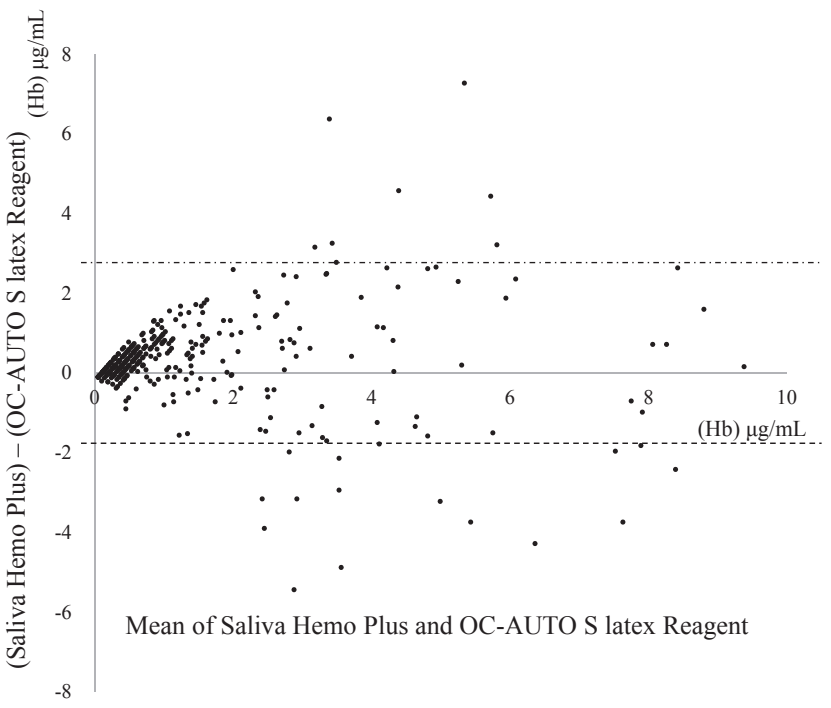

Fig. 2 Bland-Altman plot for samples with salivary $\mathrm{Hb}$ levels $<10 \mu \mathrm{g} / \mathrm{mL}$. A total of 393 sample $(70.0 \%)$ with salivary Hb levels $<10 \mu \mathrm{g} / \mathrm{mL}$ were analyzed using Bland-Altman analysis. Mean value and $95 \%$ confidence interval $(\mathrm{CI})$ of the difference was 0.29 (95\% CI\%: 0.18-0.40) and $P$ value was 0.869 when calculated using the test of no correlation. The differences by both reagents included fixed errors and no random errors. The dotted line indicates $95 \%$ CIs of the differences. Hb: hemoglobin.

S Latex Reagent, respectively. The cross tabulations for samples with $<2.0$ and $\geq 2.0 \mu \mathrm{g} / \mathrm{mL}$ and $<1.45$ and $\geq 1.45$ $\mu \mathrm{g} / \mathrm{mL}$ are summarized in Table 2 . The crude hit rates were $>95 \%$.

Circadian variations and fluctuations during the day were measured in samples from 10 subjects. The results are presented in Fig. 4. Friedman test revealed no statistically significant differences. However, on conducting Bland-Altman analysis, fixed errors were observed between wake-up time and before dinner, and before lunch and before dinner, but no random errors
Table 2 Cross tabulation of positive and negative test results obtained at 1 and 3 days after sampling

\begin{tabular}{|c|c|c|c|c|}
\hline & & \multicolumn{2}{|c|}{ Saliva Hemo Plus } & \multirow{3}{*}{$P$} \\
\hline & & \multicolumn{2}{|c|}{3 days } & \\
\hline & & $<2.0 \mu \mathrm{g} / \mathrm{mL}$ & $\geq 2.0 \mu \mathrm{g} / \mathrm{mL}$ & \\
\hline \multirow[t]{2}{*}{1 day } & $<2.0 \mu \mathrm{g} / \mathrm{mL}$ & 38 & 0 & \multirow[t]{2}{*}{$<0.001$} \\
\hline & $\geq 2.0 \mu \mathrm{g} / \mathrm{mL}$ & 1 & 23 & \\
\hline \multicolumn{5}{|l|}{ (B) } \\
\hline & & \multicolumn{2}{|c|}{ OC-AUTO S Latex Reagent } & \multirow{3}{*}{$P$} \\
\hline & & & & \\
\hline & & $<2.0 \mu \mathrm{g} / \mathrm{mL}$ & $\geq 2.0 \mu \mathrm{g} / \mathrm{mL}$ & \\
\hline \multirow[t]{2}{*}{1 day } & $<2.0 \mu \mathrm{g} / \mathrm{mL}$ & 37 & 0 & \multirow[t]{2}{*}{$<0.001$} \\
\hline & $\geq 2.0 \mu \mathrm{g} / \mathrm{mL}$ & 1 & 24 & \\
\hline \multicolumn{5}{|l|}{ (C) } \\
\hline & & \multicolumn{2}{|c|}{ OC-AUTO S Latex Reagent } & \multirow{3}{*}{$P$} \\
\hline & & & & \\
\hline & & $<1.45 \mu \mathrm{g} / \mathrm{mL}$ & $\geq 1.45 \mu \mathrm{g} / \mathrm{mL}$ & \\
\hline \multirow{2}{*}{1 day } & $<1.45 \mu \mathrm{g} / \mathrm{mL}$ & 35 & 0 & \multirow{2}{*}{$<0.001$} \\
\hline & $\geq 1.45 \mu \mathrm{g} / \mathrm{mL}$ & 2 & 25 & \\
\hline
\end{tabular}

Within 3 days, limited effects of the degradation of the saliva samples were observed for periodontitis screening if the samples were kept in the specific diluent containing preservatives. $P$ values were calculated using Fisher's exact test.

were observed (Table 3). The higher values tended to be obtained in the morning.

\section{Discussion}

Screening for periodontal diseases should be simple, easy, and cost-effective; in addition, samples must be collected using noninvasive techniques. The Community Periodontal Index (CPI), which is a traditional screening method used for periodontal diseases and involves the use of periodontal probing, may be uncomfortable or even painful for certain subjects. Although CPI was designed 


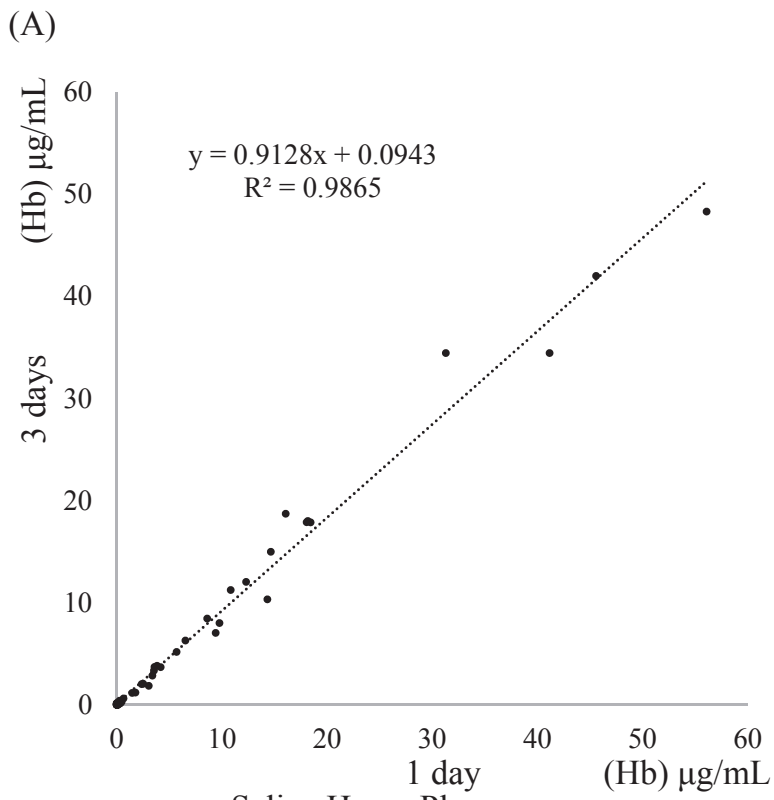

Saliva Hemo Plus

(B)

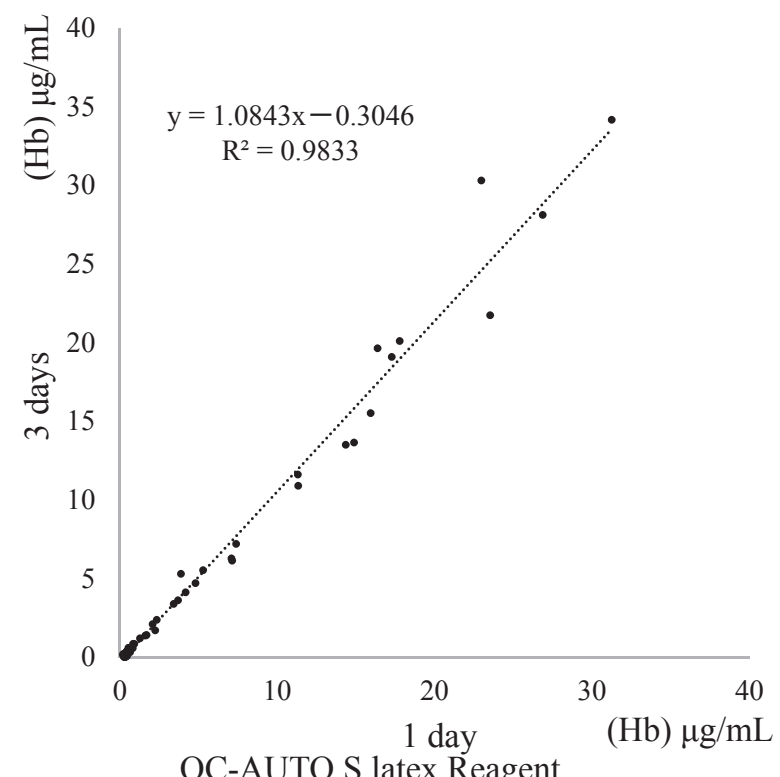

Fig. 3 Stability of salivary $\mathrm{Hb}$ levels: salivary $\mathrm{Hb}$ within 3 days. A) Saliva Hemo Plus. B) OC-AUTO S Latex Reagent. Figures present the scatter plot of salivary $\mathrm{Hb}$ levels measured at 1 and 3 days after sampling. Pearson's correlation coefficients were 0.993 for Saliva Hemo Plus and 0.992 for OC-AUTO S Latex Reagent. Moreover, the regression formulas were $(3$ days $)=1.084 \times(1$ day $)-1.451$ for Saliva Hemo Plus and $(3$ days $)=0.913 \times(1$ day $)$ +0.449 for OC-AUTO S Latex Reagent. Hb: hemoglobin.

for use in large-scale populations, the personnel cost of a trained dentist is a major issue. On the other hand, training and calibration of the dentists or examiners for salivary $\mathrm{Hb}$ tests is minimal. Moreover, salivary $\mathrm{Hb}$ tests can be simultaneously used in testing several subjects.

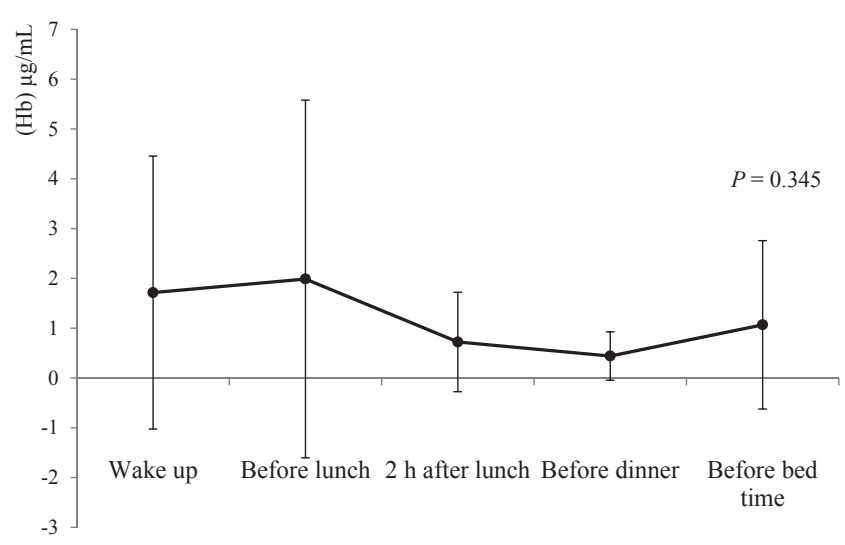

Fig. 4 Circadian variations and fluctuations during the day for salivary $\mathrm{Hb}$ levels. $P$ value was calculated using the Friedman test. Hb: hemoglobin.

The salivary $\mathrm{Hb}$ tests meet the criteria of simple, easy, and cost-effective, and are expected to become the alternative screening method to CPI.

Three salivary $\mathrm{Hb}$ tests are currently used as in vitro diagnostic methods for screening of periodontal disease and are approved under the pharmaceutical affairs law in Japan. The two methods that are the subject of this study, Saliva Hemo Plus and OC-AUTO S Latex Reagent, provide quantitative and comparative results. The third method is a qualitative colormetric method that immunologically detects human salivary $\mathrm{Hb}$ by means of a colloidal gold-labeled antihuman $\mathrm{Hb}$ polyclonal antibody (18). Therefore, in this study a comparison with the Perioscreen test (Sunstar, Osaka, Japan) was not performed.

From the scatter plots in Fig. 1, results obtained by Saliva Hemo Plus showed a strong correlation with those obtained by OC-AUTO S Latex Reagent; this suggests that both Saliva Hemo Plus and OC-AUTO S Latex Reagent can be reliably used for screening of periodontal disease. However, the salivary $\mathrm{Hb}$ levels measured by OC-AUTO S Latex Reagent tended to be lower than those measured by Saliva Hemo Plus. The Bland-Altman plot indicated that the difference included fixed errors and not random errors (Fig. 2). The fixed errors may originate from several factors. First, the two reagents used different antibody reactions; Saliva Hemo Plus uses colloidal gold agglutination reaction and OC-AUTO S Latex Reagent uses latex agglutination reaction. Second, certain components in saliva may affect the agglutination reaction. The standard curve for evaluation of a standard material is dependent on the measuring device as well as on the reagents. 
Table 3 Results of Bland-Altman analysis for circadian variations of salivary hemoglobin (Hb) levels

\begin{tabular}{|c|c|c|c|c|c|}
\hline & & Before lunch & $2 \mathrm{~h}$ after lunch & Before dinner & Before bedtime \\
\hline \multirow[t]{3}{*}{ Wake up } & Difference & -0.373 & 0.922 & $1.274 *$ & 0.648 \\
\hline & $(95 \% \mathrm{CI})$ & $(-1.451-0.908)$ & $(-0.216-2.220)$ & $(0.101-2.447)$ & $(-0.571-1.864)$ \\
\hline & $P$ value & 0.254 & 0.151 & 0.168 & 0.056 \\
\hline \multirow[t]{3}{*}{ Before lunch } & Difference & & 1.256 & $1.574 *$ & 0.921 \\
\hline & $(95 \% \mathrm{CI})$ & & $(-0.045-2.575)$ & $(0.225-2.868)$ & $(-0.186-2.028)$ \\
\hline & $P$ value & & 0.070 & 0.072 & 0.100 \\
\hline \multirow[t]{3}{*}{$2 \mathrm{~h}$ after lunch } & Difference & & & 0.282 & -0.344 \\
\hline & $(95 \% \mathrm{CI})$ & & & $(-0.343-0.908)$ & $(-1.171-0.484)$ \\
\hline & $P$ value & & & 0.111 & 0.099 \\
\hline \multirow[t]{3}{*}{ Before dinner } & Difference & & & & -0.626 \\
\hline & $(95 \% \mathrm{CI})$ & & & & $(-1.476-0.224)$ \\
\hline & $P$ value & & & & 0.180 \\
\hline
\end{tabular}

$P$ values for correlation between mean values in and differences of, salivary $\mathrm{Hb}$ levels $(\mu \mathrm{g} / \mathrm{mL}) . P$ values were calculated using the test of no correlation. $*$ indicates statistical significance. Fixed errors were observed between wake-up time and before dinner and before lunch and before dinner. There were no $P$ values $<0.05$. Therefore, there were no fixed errors.

The results of these experiments provided information that circadian variations may influence salivary $\mathrm{Hb}$ levels, while the stability of salivary $\mathrm{Hb}$ may not affect salivary $\mathrm{Hb}$ levels. Figure 4 and Table 3 present circadian variations and fluctuations during the day in salivary $\mathrm{Hb}$ levels of 10 subjects. Saliva samples from nine periodontally healthy subjects and one with periodontitis who was treated in a dental office, were evaluated. In the periodontally healthy subjects, except for one subject, higher values tended to be obtained in the morning; however, the salivary $\mathrm{Hb}$ levels were in the reference range. One periodontally healthy subject and one subject with periodontitis revealed levels that were over the reference range in the morning. The differences were not statistically significant during the day by Friedman test; this may be because the small sample size and the high standard deviation affected the results. However, higher values tended to be obtained in the morning compared with before dinner by the Bland-Altman analysis (Table 3 ). Hence, sampling time for each subject is critical as circadian variations may affect salivary $\mathrm{Hb}$ levels.

In this study, salivary $\mathrm{Hb}$ levels measured by OC-AUTO S Latex Reagent tended to be lower than those measured by Saliva Hemo Plus. Higher values tended to be observed in the morning. These two issues must be taken into consideration to promote widespread use of salivary $\mathrm{Hb}$ testing.

\section{Acknowledgments}

This work was supported by a grant from the 8020 Promotion Foundation, Japan. We would like to express our gratitude to Dr. Erika Kakuta, Dr. Hidenori Yamada, and Dr. Takatoshi Murata, Department of Translational Research, School of Dental Medicine, Tsurumi University, for their valuable advice.

\section{Conflict of interest}

The authors have no conflict of interest to declare.

\section{References}

1. Daly CG, Mitchell DH, Highfield JE, Grossberg DE, Stewart D (2001) Bacteremia due to periodontal probing: a clinical and microbiological investigation. J Periodontol 72, 210-214.

2. Spielmann N, Wong DT (2011) Saliva: diagnostics and therapeutic perspectives. Oral Dis 17, 345-354.

3. Giannobile WV (2012) Salivary diagnostics for periodontal diseases. J Am Dent Assoc 143, 6S-11S.

4. Nomura Y, Tamaki Y, Tanaka T, Arakawa H, Tsurumoto A, Kirimura $\mathrm{K}$ et al. (2006) Screening of periodontitis with salivary enzyme tests. J Oral Sci 48, 177-183.

5. Yoshie H, Tai H, Kobayashi T, Oda-Gou E, Nomura Y, Numabe $Y$ et al. (2007) Salivary enzyme levels after scaling and interleukin-1 genotypes in Japanese patients with chronic periodontitis. J Periodontol 78, 498-503.

6. Nomura Y, Shimada Y, Hanada N, Numabe Y, Kamoi K, Sato $\mathrm{T}$ et al. (2012) Salivary biomarkers for predicting the progression of chronic periodontitis. Arch Oral Biol 57, 413-420.

7. Shimazaki Y, Akifusa S, Takeshita T, Shibata Y, Doi Y, Hata J et al. (2011) Effectiveness of the salivary occult blood test as a screening method for periodontal status. J Periodontol 82, 581-587.

8. Nomura Y, Tamaki Y, Eto A, Kakuta E, Ogino D, Nakamura $\mathrm{Y}$ et al. (2012) Screening for periodontal diseases using salivary lactate dehydrogenase, hemoglobin level, and statistical modeling. J Dent Sci 7, 379-383.

9. Kakuta E, Nomura Y, Naono Y, Koresawa K, Shimizu K, Hanada N (2013) Correlation between health-care costs and salivary tests. Int Dent J 63, 249-253.

10. Nomura Y, Okada A, Kakuta E, Gunji T, Kajiura S, Hanada N (2016) A new screening method for periodontitis: an alternative to the community periodontal index. BMC Oral Health 16, 64, doi: 10.1186/s12903-016-0216-x.

11. Bland JM, Altman DG (1986) Statistical methods for assessing agreement between two methods of clinical measurement. 
Lancet 1, 307-310.

12. Bland JM, Altman DG (1995) Comparing methods of measurement: why plotting difference against standard method is misleading. Lancet 346, 1085-1087.

13. Ludbrook J (2002) Statistical techniques for comparing measurers and methods of measurement: a critical review. Clin Exp Pharmacol Physiol 29, 527-536.

14. Shimada K, Kario K, Kushiro T, Teramukai S, Ishikawa Y, Kobayashi F et al. (2015) Differences between clinic blood pressure and morning home blood pressure, as shown by Bland-Altman plots, in a large observational study (HONEST study). Hypertens Res 38, 876-882.

15. Giavarina D (2015) Understanding Bland Altman analysis. Biochem Med (Zagreb) 25, 141-151.
16. Francq BG, Govaerts B (2016) How to regress and predict in a Bland-Altman plot? Review and contribution based on tolerance intervals and correlated-errors-in-variables models. Stat Med 35, 2328-2358.

17. Gómez-Polo C, Portillo Muñoz M, Lorenzo Luengo MC, Vicente P, Galindo P, Martín Casado AM (2016) Comparison of two color-difference formulas using the Bland-Altman approach based on natural tooth color space. J Prosthet Dent 115, 482-488.

18. Pham TA, Ueno M, Shinada K, Yanagisawa T, Wright FA, Kawaguchi Y (2011) Periodontal disease and related factors among Vietnamese dental patients. Oral Health Prev Dent 9, 185-194. 